Annales Academiæ Scientiarum Fennicæ

Mathematica

Volumen 35, 2010, 389-404

\title{
TREE-LIKE DECOMPOSITIONS AND CONFORMAL MAPS
}

\author{
Christopher J. Bishop \\ SUNY at Stony Brook, Department of Mathematics \\ Stony Brook, NY 11794-3651, U.S.A.; bishop@math.sunysb.edu
}

\begin{abstract}
Any simply connected rectifiable domain $\Omega$ can be decomposed into uniformly chord-arc subdomains using only crosscuts of the domain. We show that such a decomposition allows one to construct a map from $\Omega$ to the disk which is close to conformal in a uniformly quasiconformal sense. This answers a question of Vavasis.
\end{abstract}

\section{Introduction}

Any simply connected plane domain $\Omega$ has a collection of crosscuts that divide it into uniformly chord-arc subdomains (a crosscut is an arc in $\Omega$ with distinct endpoints on $\partial \Omega$ ). We call this a tree-like decomposition of $\Omega$ since the pieces form the vertices of a tree under the obvious adjacency relation. Vavasis suggested using tree-like decompositions to approximate the Riemann map from $\Omega$ to the unit disk, $\mathbf{D}$, and here we make this idea precise by associating to such a decomposition a map $\partial \Omega \rightarrow$ $\mathbf{T}=\partial \mathbf{D}$ that has a uniformly quasiconformal extension to the interiors.

If $\Omega$ has a rectifiable boundary then the most obvious map of $\partial \Omega$ to a circle is the one which preserves length up to a constant factor. If the boundary is also chord-arc, then this map is known to have an quasiconformal extension to a map $\Omega \rightarrow \mathbf{D}$ with constant depending only on the chord-arc constant of $\partial \Omega$. Given a domain with a tree-like decomposition into rectifiable pieces we will define a "piecewise length preserving map" and if the pieces are chord-arc with uniformly bounded constant, we will show this boundary map also has a uniformly QC extension to the interior.

So suppose we are given a tree-like decomposition $\left\{\Omega_{k}\right\}$ of the domain $\Omega$ into rectifiable pieces. Define the "obvious" map on each piece and glue them together as follows: (1) map $f_{k}: \partial \Omega_{k} \rightarrow \mathbf{T}$ by a map multiplying length by a constant factor, (2) map $\mathbf{T}$ to the hyperbolic convex hull of $E_{k}=f\left(\partial \Omega_{k} \cap \partial \Omega\right)$ by the hyperbolic nearest point retraction $N_{k}$ and (3) apply a Möbius transformation $\tau_{k}$ of the disk so the image "matches up" with the image of its parent (i.e., the maps for a piece and its parent are normalized to agree at the endpoints and center of the crosscut separating them; at other points along the crosscut the maps may differ, but this will not be important and we will show later the discontinuity is bounded in the hyperbolic metric). Let $\psi_{k}=\tau_{k} \circ N_{k} \circ f_{k}: \partial \Omega_{k} \rightarrow \overline{\mathbf{D}}$ and define $\psi: \partial \Omega \rightarrow \mathbf{T}$ by setting $\psi=\psi_{k}$ on $\partial \Omega_{k} \cap \partial \Omega$. We will call this a piecewise length preserving map (or PLP map).

doi:10.5186/aasfm.2010.3525

2000 Mathematics Subject Classification: Primary 30C35; Secondary 30C30, 65E05, 30C62.

Key words: Conformal mapping, quasiconformal maps, inner chord-arc domains, numerical conformal mapping, hyperbolic geometry, Schwarz-Christoffel formula.

The author is partially supported by NSF Grant DMS 10-06309. 


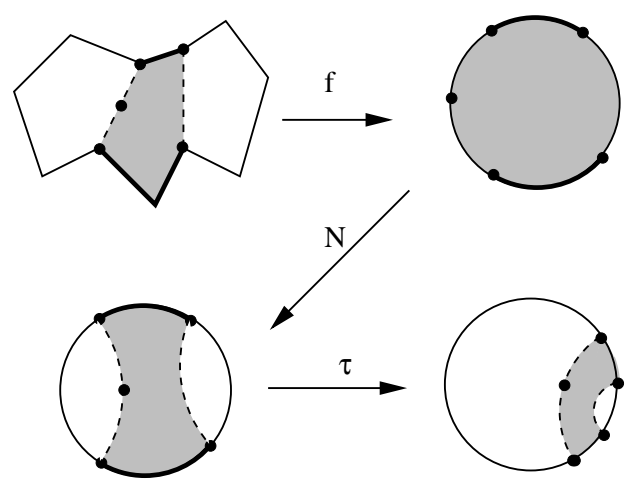

Figure 1. The boundary of each piece of the decomposition is mapped into the disk by a composition of three maps: a length preserving map $f$, the retraction to a hyperbolic convex hull $N$ and a Möbius transformation $\tau$ to match it against previously defined maps.

Theorem 1.1. Suppose $\Omega$ is a simply connected domain with a tree-like decomposition into chord-arc pieces, all with constants $\leq M$. Then the PLP map defined above has a quasiconformal extension $\Psi: \Omega \rightarrow \mathbf{D}$ with $Q C$ constant depending only of $M$.

Actually we will prove this under the weaker assumption that the decomposition pieces are inner chord-arc (both chord-arc and inner chord-arc domains will be defined in Section 3). Explicit maps that are approximately conformal are used in various computational conformal mapping techniques such as the CRDT algorithm of Driscoll and Vavasis [11], [4]), the medial axis method in [5], [7] and Davis' method [9]. In fact, we can think of our map as a way of making Davis' method behave like CRDT for general domains. See Section 5 .

The map $N_{n}$ is not really needed in the definition above; in fact, since it is the identity on $E_{n}$, it does not effect the values of final map except through the choice of $\tau$, and this could have been accomplished in other ways, e.g. using cross ratios as in CRDT. The advantage of introducing $N_{n}$ really concerns the proof of Theorem 1.1, rather than its statement. Using the retraction map sends pieces of the decomposition to non-overlapping sets in $\mathbf{D}$ and defines a map of $\Omega \rightarrow \mathbf{D}$. This map need not be quasiconformal (see Figure 2), but we shall prove that it is a quasiisometry between the hyperbolic metrics of $\Omega$ and $\mathbf{D}$ and hence (by known results) there is a quasiconformal map with the same boundary values.
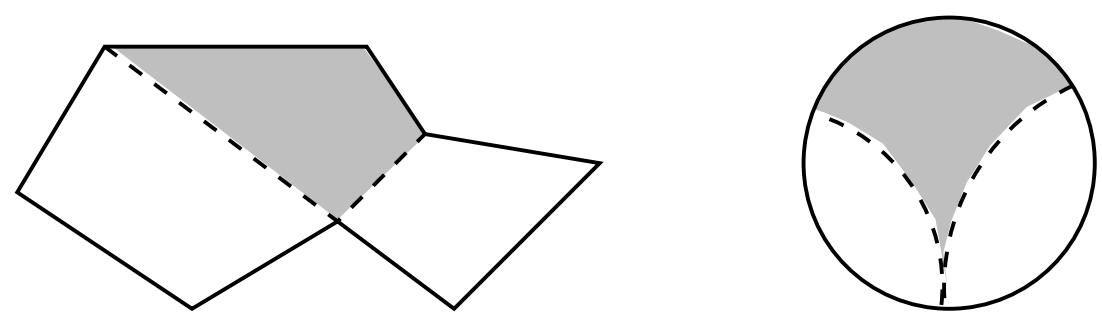

Figure 2. The extension to the interior we have described is not quasiconformal in general. For example, two crosscuts that have a common endpoint can be a positive hyperbolic distance apart in $\Omega$, but be mapped to geodesics in $\mathbf{D}$ which are distance 0 apart in $\mathbf{D}$. This is impossible for a quasiconformal map, but possible for our map which is only a quasi-isometry. 
This paper is one of three related papers that were prompted by questions of Vavasis. He was interested in whether nice tree-like decompositions of a domain exist, and whether they could be used to construct a good approximation of the conformal map. He also suggested using such a decomposition to study harmonic conjugation on $L^{2}(\partial \Omega)$. In [8], we answer his first question affirmatively and here we answer the second. In [6] we estimate the $L^{2}$ norm of harmonic conjugation using tree-like decompositions.

I thank Stephen Vavasis for his comments on earlier drafts of this paper. I also thank the referee for numerous comments and suggestions that greatly improved the clarity of the paper.

In Section 2 we review some basic facts about hyperbolic geometry, conformal and quasiconformal maps. In Section 3 we prove a few results about tree-like decompositions and in Section 4 we prove Theorem 1.1. In Section 5 we conclude with an example of how our map could be used in the numerical approximation of conformal maps and compare this to CRDT.

\section{Hyperbolic geometry and quasiconformal maps}

The hyperbolic metric on the unit disk is given locally by

$$
|d \rho|=\frac{2|d z|}{1-|z|^{2}} .
$$

Hyperbolic geodesics are circular arcs which are orthogonal to the boundary. Möbius transformations of the disk are isometries for the hyperbolic metric.

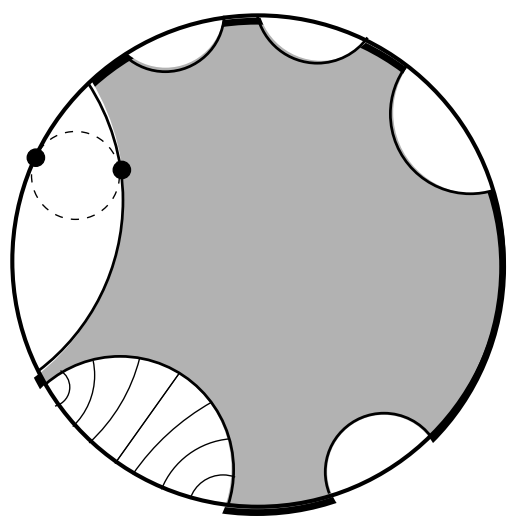

Figure 3. The hyperbolic convex hull of a closed set on the circle. The nearest point retraction collapses circular arcs foliating the complementary crescents. For points $z$ on the unit circle we can visualize this map by expanding a ball tangent to $\mathbf{T}$ at $z$ until it first makes contact with the convex hull.

Suppose $E \subset \mathbf{T}$ is compact. Then $\mathbf{T} \backslash E=\cup_{k} I_{k}$ is a union of open intervals. Corresponding to each $I_{k}$ there is a hyperbolic geodesic with the same endpoints. These geodesics, together with $E$, bound a region in $\mathbf{D}$ called the hyperbolic convex hull of $E$ and denoted by $C(E)$. The complement of $C(E)$ in $\mathbf{D}$ is a union of crescents, each bounded by $I_{k} \cup \gamma_{k}$. Each crescent has an foliation by circular arcs orthogonal to both boundary arcs and following these arcs until they hit $\gamma_{k}$ defines a continuous map of the crescent onto $\gamma_{k}$. If we extend this map to all of $\mathbf{D}$ by letting it be 
the identity on $C(E)$, we get a map $\mathbf{D} \rightarrow C(E)$ which is called the nearest point retraction (because it corresponds to mapping each point of $\mathbf{D}$ to the unique closest point of $C(E)$ in the hyperbolic metric).

We will need the following estimate regarding hyperbolic geodesics.

Lemma 2.1. Suppose $z_{1}, z_{2}, w_{1}, w_{2} \in \mathbf{D}$ and $\rho\left(z_{1}, z_{2}\right) \leq A, \rho\left(w_{1}, w_{2}\right) \leq A$ and $\rho\left(z_{1}, w_{1}\right) \geq B$. Let $\gamma_{k}$ be the hyperbolic geodesic connecting $z_{k}$ to $w_{k}$ for $k=1,2$. Let $x$ be the midpoint of $\gamma_{1}$. Then $\gamma_{2}$ passes within hyperbolic distance $C \exp \left(A-\frac{1}{2} B\right)$ of $x$.

Proof. We may assume $x=0$, and $\gamma_{1}$ is on the real axis. Thus $z_{1}, w_{1}$ will be points on the real axis approximately Euclidean distance $e^{-B / 2}$ from $+1,-1$ respectively. Thus $z_{2}, w_{2}$ lie in disks of Euclidean radius $\simeq \exp (A-B / 2)$ around \pm 1 . Thus the geodesic between them passes within $O\left(\exp \left(A-\frac{1}{2} B\right)\right)$ of the origin as desired.

Simply connected, proper subdomains of the plane inherit a hyperbolic metric from the unit disk via the Riemann map. If $\varphi: \mathbf{D} \rightarrow \Omega$ is conformal and $w=\varphi(z)$ then $\rho_{\Omega}\left(w_{1}, w_{2}\right)=\rho_{\mathbf{D}}\left(z_{1}, z_{2}\right)$ defines the hyperbolic metric on $\Omega$ and is independent of the particular choice of $\varphi$. It is often convenient to estimate $\rho_{\Omega}$ in terms of the more geometric quasi-hyperbolic metric on $\Omega$ which is defined as

$$
\tilde{\rho}\left(w_{1}, w_{2}\right)=\inf \int_{w_{1}}^{w_{2}} \frac{|d w|}{\operatorname{dist}(w, \partial \Omega)},
$$

where the infimum is over all arcs in $\Omega$ joining $w_{1}$ to $w_{2}$. It follows from Koebe's $\frac{1}{4}$ theorem that the two metrics are comparable with bounds independent of the domain. A Whitney decomposition of a domain $\Omega$ is a covering of $\Omega$ by squares $\left\{Q_{k}\right\}$ with disjoint interiors and the property that $\operatorname{diam}\left(Q_{k}\right) \simeq \operatorname{dist}\left(Q_{k}, \partial \Omega\right)$. By our remarks above, each square in a Whitney decomposition has uniformly bounded hyperbolic diameter (and contains a ball with hyperbolic radius bounded uniformly from below). Thus bounding the hyperbolic length of a path often reduces to simply estimating the number of Whitney squares it hits. Here is a simple but useful estimate of the hyperbolic distance. Let $d(z)=\operatorname{dist}(z, \partial \Omega)$ (Euclidean distance).

Lemma 2.2. Suppose $\Omega$ is simply connected and $z, w \in \Omega$. If $|z-w| \geq d(w) \geq$ $2 d(z)$, then

$$
\rho(z, w) \gtrsim \log \frac{d(w)}{d(z)} .
$$

If $|z-w| \geq \max (d(z), d(w))$, then

$$
\rho(z, w) \gtrsim \log \frac{|z-w|}{d(z)}+\log \frac{|z-w|}{d(w)} .
$$

If, in addition, there is an $\epsilon>0$ and a curve $\sigma$ in $\Omega$ from $z, w$ with the property that $d(x) \geq \epsilon \cdot \operatorname{dist}(x,\{z, w\})$ for every $x \in \sigma$, then

$$
\rho(z, w) \simeq \log \frac{|z-w|}{d(z)}+\log \frac{|z-w|}{d(w)},
$$

with a constant depending on $\epsilon$ (at most $O\left(\epsilon^{-2}\right)$ ).

Proof. It is enough to prove this for the quasi-hyperbolic metric, since it is boundedly equivalent to the hyperbolic metric. Assume $d(z) \leq d(w)$. Let $A_{n}$ be the 
annulus

$$
A_{n}=\left\{x: 2^{n} d(z)<|z-x|<2^{n+1} d(z)\right\},
$$

and let $B_{n}$ be the corresponding annuli around $w$. At each point of $A_{n}$ the distance to the boundary of $\Omega$ is $\lesssim \operatorname{diam}\left(A_{n}\right)$, so any curve that crosses the annulus has hyperbolic length bounded uniformly from below. We can fit $\simeq \log d(w) / d(z)$ disjoint annuli between $z$ and $w$ so, this gives a lower bound for the hyperbolic distance between them.

If $|z-w| \geq \max (d(z), d(w))$ then we can fit $\simeq \log |z-w| / d(z)$ annuli around the point $z$ and inside the disk $D(z,|z-w| / 2)$. Similarly we can fit $\simeq \log |z-w| / d(w)$ annuli around $w$ and inside $D(w,|z-w| / 2)$. Thus the sum of these numbers is a lower bound for the hyperbolic distance in this case.

If there is a curve between the points with the given bound, then this curve can be covered by Whitney squares for $\Omega$. See Figure 4. Only a uniformly bounded number of such squares of a fixed size can hit an annulus of comparable size, so the total number of distinct squares hit by the curve is bounded by a multiple of the number of annuli. Each Whitney square has uniformly bounded hyperbolic diameter, and they connect $z$ to $w$, so the hyperbolic distance between $z$ and $w$ is bounded by multiple of the number of squares, and hence the number of annuli. This is the claimed estimate.

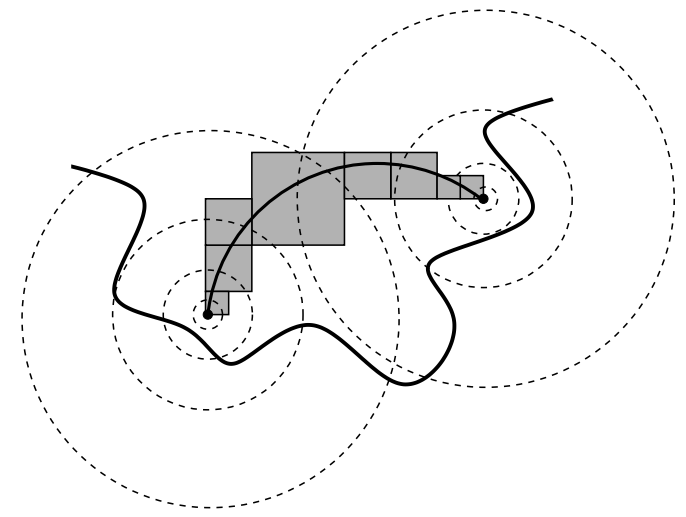

Figure 4. Estimating the hyperbolic distance between points, by counting number of Whitney squares needed to connect them.

If the given curve $\sigma$ has the property that its intersection with any Whitney cube has (Euclidean) length bounded by a multiple of the cube's diameter, then the proof above shows the hyperbolic length of $\sigma$ is bounded by the hyperbolic distance between $z$ and $w$. We will use this observation later.

There are several equivalent definitions of a $K$-quasiconformal mapping between planar domains. Suppose $f: \Omega \rightarrow \Omega^{\prime}$ is a homeomorphism. We say $f$ is $K$-quasiconformal if either of the following equivalent conditions holds:

Analytic definition: $f$ is absolutely continuous on almost every vertical and horizontal line and the partial derivatives of $f$ satisfy $\left|f_{\bar{z}}\right| \leq k\left|f_{z}\right|$ where $k=(K-1) /(K+1)$.

Metric definition: For every $x \in \Omega$

$$
\limsup _{r \rightarrow 0} \frac{\max _{y:|x-y|=r}|f(x)-f(y)|}{\min _{y:|x-y|=r}|f(x)-f(y)|} \leq K .
$$


Quasiconformal maps generalize biLipschitz maps, i.e., maps that satisfy

$$
\frac{1}{K} \leq \frac{|f(x)-f(y)|}{|x-y|} \leq K .
$$

From the metric definition it is clear that any $K$-biLipschitz map is $K^{2}$-quasiconformal. Although a quasiconformal map $f: \mathbf{D} \rightarrow \mathbf{D}$ need not be biLipschitz, it is a quasiisometry of the disk with its hyperbolic metric $\rho$, i.e., there is a constant $A$ such that

$$
\frac{1}{A} \rho(x, y)-B \leq \rho(f(x), f(y)) \leq A \rho(x, y)+B .
$$

This says $f$ is biLipschitz for the hyperbolic metric at large scales. Note that to show a map $f$ is a quasi-isometry it suffices to show that both $f(E)$ and $f^{-1}(E)=$ $\{x: f(x) \in E\}$ have uniformly bounded diameter whenever $E$ is a set of diameter 1 (assuming any two points in the domain and image space can be connected by a curve with length comparable to distance between the points, which holds in the cases we will consider).

A boundary mapping $f: \mathbf{T} \rightarrow \mathbf{T}$ is a quasisymmetric homeomorphism if there is an $k<\infty$ (depending only on $K$ ) so that $1 / k \leq|f(I)| /|f(J)| \leq k$, whenever $I, J \subset \mathbf{T}$ are adjacent intervals of equal length. It turns out that all the classes discussed above have the same set of boundary values and these are all given by the quasisymmetric maps, i.e.,

Theorem 2.3. For a map $f: \mathbf{D} \rightarrow \mathbf{D}$ we have $(1) \Rightarrow(2) \Rightarrow(3) \Rightarrow(4)$ where

(1) $f$ is biLipschitz with respect to the hyperbolic metric.

(2) $f$ is quasiconformal.

(3) $f$ is a quasi-isometry with respect to the hyperbolic metric.

(4) $f$ has a continuous extension to $\mathbf{T}$ which is quasisymmetric.

Moreover, any quasisymmetric homeomorphism of the circle has a continuous extension to the disk which satisfies (1).

The implication $(1) \Rightarrow(2)$ is clear from the definitions. $(2) \Rightarrow(3)$ is proven in [12] by Epstein, Marden and Markovic with $A=K$ and $B=K \log 2$ if $1 \leq K \leq 2$ and $B=2.37(K-1)$ if $K>2$. (3) $\Rightarrow(4)$ is a result of of Väisälä [18]. (4) $\Rightarrow(1)$ is a standard fact; see e.g. Ahlfors' book [1].

\section{Hyperbolic geometry of chord-arc decompositions}

Given a set $E$ in the plane we define its 1-dimensional measure as

$$
\ell(E)=\lim _{\delta \rightarrow 0} \inf \left\{\sum 2 r_{j}: E \subset \cup B\left(x_{j}, r_{j}\right), r_{j} \leq \delta\right\}
$$

where the infimum is over all covers of $E$ by open balls. We denote it by $\ell(E)$, since if $E$ is a Jordan curve, this agrees with the usual notion of length. We say that a simply connected domain $\Omega$ has a rectifiable boundary if $\ell(\partial \Omega)<\infty$. A Jordan domain is called chord-arc if there is an $M<\infty$ so that

$$
\ell(\sigma(x, y)) \leq M|x-y|
$$

for all $x, y \in \partial \Omega$, where $\sigma(x, y)$ denotes the shorter arc on $\partial \Omega$ between these points. If $\Omega$ is chord-arc then a map $f: \partial \Omega \rightarrow \partial \mathbf{D}$ that multiplies length by $2 \pi / \ell(\partial \Omega)$ has a quasiconformal extension to the interiors with a constant depending only on the 
chord-arc constant of $\Omega$ (e.g., this follows from Theorem VII.4.3 of [13]). In fact, this holds for "inner chord-arc" defined as follows.

Any crosscut $\gamma$ of $\Omega$ is the conformal image of a crosscut in $\mathbf{D}$ which defines two $\operatorname{arcs}$ on $\mathbf{T}$. We will say $\Omega$ is inner chord-arc with constant $M$ if for one of these arcs $I$, we have

$$
\int_{I}\left|f^{\prime}\right||d z| \leq M \ell(\gamma)
$$

In other words, $I$ has length bounded by a multiple of any crosscut with the same endpoints. It is a theorem of Gehring and Hayman [14] that all such crosscuts have length bounded by a multiple of the Euclidean length of the hyperbolic geodesic with the same endpoints, so we need only consider geodesics in this definition. Inner chordarc domains were introduced by Pommerenke in [16] and also studied by Väisälä in [17] and Ghamsari [15]. They generalize the usual notion of chord-arc domain, where the boundary is a Jordan curve and the length of the shortest boundary arc connecting $x, y$ is bounded by $M|x-y|$.

A curve $\gamma$ is called regular if there is an $M<\infty$ so that the length of $\gamma \cap D$ is bounded by $M r$ for every disk $D=D(x, r)$. It is not hard to see that the boundary of a inner chord-arc domain $\Omega$ must be regular. There is nothing to do if $r \simeq \operatorname{diam}(\Omega)$, so assume $r \ll \operatorname{diam}(\Omega)$, Choose a base point $z \in \Omega \backslash D$ with $\operatorname{dist}(z, \partial \Omega)>r$. The part of $\gamma$ inside $D$ is separated from $z$ by arcs of $\partial D$ and by the inner chord-arc condition their length is bounded by a multiple of the lengths of these arcs (which are crosscuts of $\Omega$ ). The total length of the arcs is at most $2 \pi r$, so we are done. See Figure 5. This is due to Ghamsari [15].

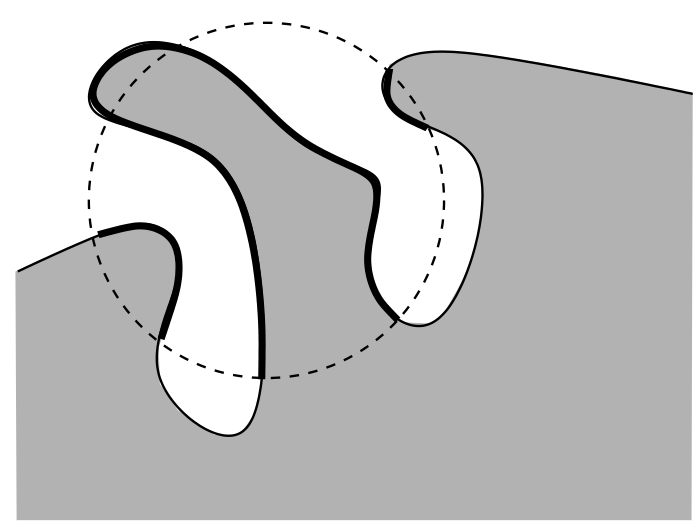

Figure 5. Proof that an inner chord-arc boundary is regular.

If two inner chord-arc domains share a common boundary arc $\gamma$, then $\gamma$ must be chord arc. To see this, choose basepoints in each domain as above and consider any two points $z, w \in \gamma$ with $|z-w|$ less than the distance of either basepoint to the boundary. Let $D=D((z+w) / 2,|z-w| / 2)$. Every point of the arc of $\gamma$ between $z$ and $w$ is separated from one of the basepoints by some arc of $\partial D$. Thus the argument above shows the length of this arc is $O(r)$, i.e., $\gamma$ is chord-arc.

If $\Omega$ is inner chord-arc, we can still define a map $f: \partial \Omega \rightarrow \partial \mathbf{D}$ which multiplies length by a constant, but we have to interpret $\partial \Omega$ as a topological circle. It is a result of Väisälä [17] that an inner chord-arc domain $\Omega$ is a locally biLipschitz image of the disk, say by a map $g: \mathbf{D} \rightarrow \Omega$. Then $f \circ g: \mathbf{T} \rightarrow \mathbf{T}$ is biLipschitz, hence quasisymmetric, hence has a quasiconformal extension $\Phi: \mathbf{D} \rightarrow \mathbf{D}$. Thus 
$\Phi \circ g^{-1}: \Omega \rightarrow \mathbf{D}$ is an extension of $f$ and is a composition of a locally biLipschitz map and a quasiconformal map, hence is quasiconformal.

In what follows we will assume $\Omega$ is decomposed into inner chord-arc pieces (but the reader may assume the pieces are chord-arc to avoid the technicalities about the boundary map described above).

Lemma 3.1. Suppose $\Omega$ is simply connected, $\Gamma=\left\{\gamma_{k}\right\}$ is a disjoint collection of crosscuts in $\Omega$ and $\Omega \backslash \Gamma=\cup_{k} \Omega_{k}$ is a tree-like decomposition of $\Omega$ into inner chord-arc domains with constant $M$. Then there is an $\epsilon>0$, depending only on $M$, so that

(1) any two crosscuts from $\Gamma$ are separated by at least hyperbolic distance $\epsilon$,

(2) For each crosscut $\gamma$ and every $z \in \gamma$, dist $(z, \partial \Omega) \geq \epsilon \operatorname{dist}(z, \partial \gamma)$, where $\partial \gamma$ denotes the two endpoints of $\gamma$.

Proof. Suppose $z \in \gamma$ and $z^{\prime} \in \gamma^{\prime}$ are points on distinct crosscuts such that $\rho\left(z, z^{\prime}\right)<\epsilon$. Without loss of generality we may assume $\gamma, \gamma^{\prime}$ are both on the boundary of the same decomposition piece $\Omega_{k}$ (otherwise consider the segment $S$ between them and replace these points by the endpoints of some component of $S \backslash \Gamma$ which are in different crosscuts, but still less than $\epsilon$ apart). Thus both arcs of $\partial \Omega_{k}$ which connect $z, z^{\prime}$ hit the boundary of $\Omega$ and hence have length $\geq d(z)=\operatorname{dist}(z, \partial \Omega)$, whereas the chord between $z$ and $z^{\prime}$ has Euclidean length $\leq \epsilon d(z)$. This is a contradiction for $\epsilon$ small, so (1) holds. A similar argument proves (2).

If $\Omega$ is simply connected we say that $E \subset \Omega$ is quasi-convex with constant $C$ if the shortest hyperbolic path in $E$ between any two points $z, w$ has length at most $C$ times the hyperbolic distance $\rho(z, w)$ in $\Omega$ (the length of a path in $E$ is measured using the hyperbolic distance on $\Omega$ restricted to $E$ ).

Lemma 3.2. Suppose $\Omega$ is simply connected, and $\left\{\Omega_{k}\right\}$ is a tree-like decomposition of $\Omega$ into inner chord-arc domains with constant $M$. Then each $\Omega_{k}$ is quasiconvex with a constant depending only on $M$. Moreover, each crosscut $\gamma$ of the decomposition is quasi-convex.

Proof. If we can show the boundary arcs are quasi-convex then the interior must be as well, since we can modify a hyperbolic geodesic in $\Omega$ between two points of $\Omega_{k}$ to follow the boundary of $\Omega_{k}$ whenever it leaves $\Omega_{k}$. So suppose $z, w \in \gamma \subset \partial \Omega_{k} \cap \Omega$ and that $\sigma$ is the arc of $\gamma$ connecting them. Then $\sigma$ is chord-arc and by our remarks following Lemma 2.2 and part (2) of Lemma 3.1, the hyperbolic length of $\sigma$ is bounded by a multiple of the hyperbolic distance between $z$ and $w$, so we are done.

Lemma 3.3. Suppose $\gamma$ is either a crosscut of $\Omega$ or connects two points $z, w$ of $\Omega$. Assume $\gamma$ is quasi-convex with constant $M$. Then there is an $A<\infty$ (depending only on $M$ ) so that $\tilde{\gamma}$ stays within a hyperbolic $A$-neighborhood of $\gamma$, where $\tilde{\gamma}$ is the hyperbolic geodesic with the same endpoints.

Proof. We prove this by assuming the conclusion fails for a large $A$, and find two points on $\gamma$ where the quasi-convexity also fails. With loss of generality, assume $\Omega$ is the upper half-plane and normalize so that $\tilde{\gamma}$ lies on the positive imaginary axis. If $\gamma$ is a crosscut then $\tilde{\gamma}$ is the whole axis. Otherwise we assume the points $z, w$ are at least distance 10 apart and that we have normalized so $\operatorname{Im}(\mathrm{w}) \geq \mathrm{e}^{5}$ and $\operatorname{Im}(\mathrm{z}) \leq \mathrm{e}^{-5}$.

Suppose that $i=\sqrt{-1}=(0,1)$ is point of $\tilde{\gamma}$ which is at least distance $C$ from $\gamma$. Then $\gamma \cap D(0,1)$ must lie below the horizontal line $\{y=\epsilon\}$ where $\epsilon=O(\exp (-C))$. 
Fix $s \in[-1,1]$ and $t>0$ and consider an open truncated cone with vertex at $s+i t$, i.e.,

$$
\Gamma(s, t)=\{z=x+i y:-1<x<1,1>y>6 \epsilon|x-s|+t\} .
$$

First suppose $s_{1}=1 / 6$ and choose the the minimal $t>0$ so that $\Gamma\left(s_{1}, t\right)$ is disjoint from $\gamma$. If this is strictly positive stop. If it is 0 , then change $s_{1}=-1 / 6$ and consider the same way of choosing $t$. If we get 0 again, then $\gamma$ can't connect 0 to $\infty$ in $\mathbf{H}$ so one of these $t$ 's must be positive. Fix $s_{1}$ to be the choice (either $\pm 1 / 6$ ) that gives a positive value for $t$ and let this value be denoted $t_{1}$. Now define $s_{2}, t_{2}$ in the same way except with $\left|s_{2}\right|=5 / 6$. See Figure 6 . Let

$$
W=\{x+i y:-1<x<1,0<y<\epsilon\} \backslash\left(\Gamma\left(s_{1}, t_{1}\right) \cup \Gamma\left(s_{2}, t_{2}\right) .\right.
$$

By the minimality of $t_{1}$ and $t_{2}$, there is a point $z_{1}=x_{1}+i y_{1} \in \gamma \cap \partial \Gamma\left(s_{1}, t_{2}\right)$ and a point $z_{2}=x_{2}+i y_{2} \in \gamma \cap \partial \Gamma\left(s_{2}, t_{2}\right)$. The hyperbolic distance between these points is

$$
\simeq\left|\log y_{1}\right|+\left|\log y_{2}\right|=|\log \epsilon|+\left|\log \epsilon / y_{1}\right|+\left|\log \epsilon / y_{2}\right|,
$$

but the length of $\gamma$ between these points is at least the length of $\partial W$ between these points (since $\partial W$ lies above $\gamma$ ) which is

$$
\geq \frac{c}{\epsilon}\left(\log \epsilon / y_{1}\right)+1 /(3 \epsilon)+\frac{c}{\epsilon}\left(\log \epsilon / y_{2}\right) .
$$

Since (3.2) 》(3.1) if $\epsilon$ is small, we get a contradiction that proves the lemma.

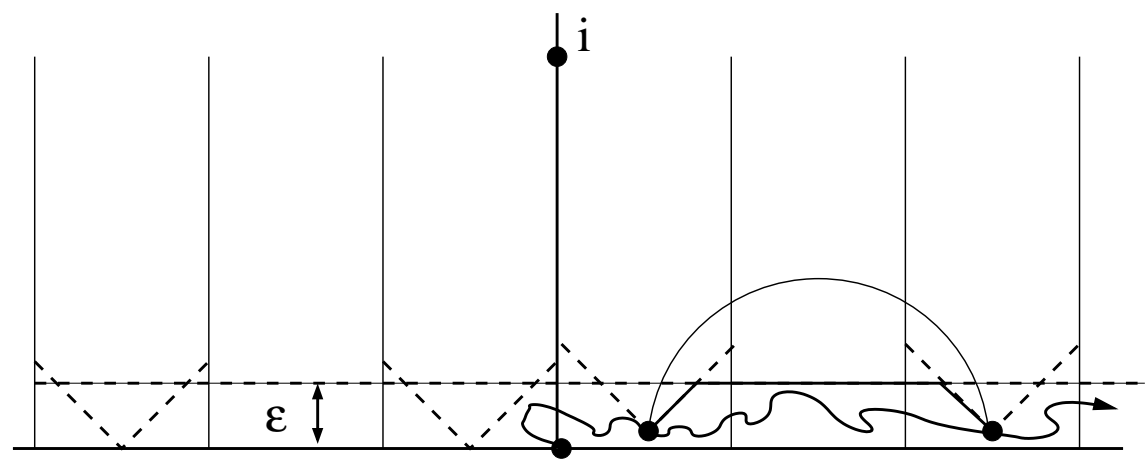

Figure 6. If $\gamma$ does not follow a geodesic it can't be quasiconvex. If $\gamma$ lies below the dashed horizontal line there must be two points whose $\gamma$ distance apart is much larger than there hyperbolic distance.

Corollary 3.4. Suppose $\Omega$ is a simply connected domain which is decomposed into inner chord-arc subdomains by a collection of crosscuts $\left\{\gamma_{k}\right\}$ and let $\left\{\tilde{\gamma}_{k}\right\}$ be the collection of hyperbolic geodesics with the same endpoints. Given a radius $r$, only a bounded number $C=C(r)$ of the $\tilde{\gamma}_{k}$ can intersect any hyperbolic ball of radius $r$.

Proof. Suppose some of the geodesics intersect a ball $B$. Then at least that number of crosscuts intersect the concentric ball of radius $r+A$ (where $A$ is from Lemma 3.3). The crosscuts are uniformly separated in the hyperbolic metric so this number is bounded depending only on $r+A$.

Lemma 3.5. Suppose $\Omega$ is simply connected, and $\left\{\Omega_{k}\right\}$ is a tree-like decomposition of $\Omega$ into inner chord-arc domains with constant $M$. Let $f_{k}: \Omega_{k} \rightarrow \mathbf{D}$ be the map described above and let $E_{k}=f_{k}\left(\partial \Omega_{k} \cap \partial \Omega\right) \subset \mathbf{T}$. Let $N_{k}$ be the nearest point 
retraction onto $C\left(E_{k}\right)$ (the hyperbolic convex hull of $E_{k}$ ). Let $\tau_{k}$ be a Möbius mapping of the disk to itself. Then $\tau_{k} \circ N_{k} \circ f_{k}$ is a quasi-isometry from the hyperbolic metric of $\Omega$ restricted to $\Omega_{k}$ to the hyperbolic metric on $\mathbf{D}$.

Proof. Since a Möbius self-mapping of the disk is an isometry of the hyperbolic metric, we may ignore $\tau_{k}$. As noted earlier, we have to show that forward and backward images of unit balls have bounded diameter. However, by the definition of our maps and Lemma 3.1, it is clear that for $z \in \Omega_{k}$

$$
\frac{\operatorname{dist}(z, \partial \Omega)}{\operatorname{diam}\left(\Omega_{k}\right)} \simeq \frac{\operatorname{dist}\left(z, \partial \Omega_{k} \cap \partial \Omega\right)}{\operatorname{diam}\left(\Omega_{k}\right)} \simeq \operatorname{dist}\left(f_{k}(z), E_{k}\right) \simeq \operatorname{dist}\left(N_{k}\left(f_{k}(z)\right), \mathbf{T}\right) .
$$

Since $\Omega_{k}$ is quasi-convex, this implies that the forward image of a unit ball intersected with $\Omega_{k}$ has bounded hyperbolic diameter in $\mathbf{D}$.

Similarly, given two points in the image which are unit distance apart, consider a curve connecting them in the interior of the image whose length is at most twice the hyperbolic distance between them. Then $N_{k}^{-1}$ of the interior is itself, and $f_{k}^{-1}$ is a quasi-isometry, so the interior has inverse image which has bounded diameter. Thus we only have to check the inverse images of the endpoints, $z, w$, and we may assume they are on the boundary of the image. Then $N_{k}^{-1}$ of a single point is a circular arc connecting the boundary of the image to the unit circle and $f_{k}^{-1}$ sends this to an arc whose Euclidean diameter and distance to $\partial \Omega$ are comparable (and hence have bounded hyperbolic diameter). This completes the proof.

Lemma 3.6. Suppose $\Omega$ is simply connected, and $\left\{\Omega_{k}\right\}$ is a tree-like decomposition of $\Omega$ into inner chord-arc domains with constant $M$. Let $f_{k}, N_{k}$ be as above. Then $N_{k} \circ f_{k}$ is biLipschitz on each crosscut (between the hyperbolic metrics on $\Omega$ and $\mathbf{D})$.

Proof. If $z \in \gamma \subset \partial \Omega_{k}$ then $N_{k} \circ f_{k}$ maps $z$ to a point $w$ with

$$
\frac{\operatorname{dist}(z, \partial \Omega)}{\operatorname{diam}(\gamma)} \simeq \frac{1-|w|}{\operatorname{diam}\left(f_{k}(\gamma)\right)}
$$

This holds because $f_{k}$ multiplies distances and so $f(z)$ is the essentially the same distance (proportionally) from the endpoints of $f(\gamma)$ that $z$ is from the endpoints of $\gamma$ (we are also using that $\gamma$ is chord-arc). Then the definition of $N_{k}$ implies that $w=N_{k}\left(f_{k}(z)\right)$ is essentially the same distance from $\partial \mathbf{D}$ that $f_{k}(z)$ was from the endpoints of $f_{k}(\gamma)$. Finally, the displayed equation implies the composed map is biLipschitz.

\section{Proof of Theorem 1.1}

Recall from the introduction that we define a map $\psi_{n}$ on each $\Omega_{n}$ in the decomposition as a composition

$$
\psi_{n}: f_{n} \circ N_{n} \circ \tau_{n}
$$

where $f_{n}$ maps $\Omega_{n}$ onto the disk and multiplies boundary length by the constant factor $2 \pi / \ell\left(\partial \Omega_{n}\right), N_{n}$ is the nearest point retraction on $C\left(E_{n}\right)$ where $E_{n}=f_{n}\left(\partial \Omega_{n} \cap \partial \Omega\right)$ and $\tau_{n}$ is a normalizing Möbius transformation so that the maps $\psi_{n}, \psi_{k}$ corresponding to adjacent pieces agree on the endpoints and midpoint of the crosscut separating $\Omega_{n}$ and $\Omega_{k}$. This defines a continuous map of $\partial \Omega$, but it not necessarily continuous on the interior. However, we will prove that it is a quasi-isometry between the hyperbolic 
metrics of $\Omega$ and $\mathbf{D}$. Thus the boundary values have some other extension to the interior which is quasiconformal.

Lemma 4.1. If $x \in \gamma_{n}=\partial \Omega_{n} \cap \partial \Omega_{k}$ then the two maps $\psi_{n}, \psi_{k}$ map $x$ to points that are $O(1)$ apart in the hyperbolic metric of $\mathbf{D}$.

Proof. This is clear since both maps send a point $z$ to images which lie on the same geodesic and have comparable distances from the unit circle.

Corollary 4.2. With notations as above, if $\Omega_{1}, \Omega_{2}$ are decompositions pieces that share a common crosscut $\gamma$ in their boundaries and $z_{1} \in \Omega_{1}, z_{2} \in \Omega_{2}$ are such that $\rho_{\mathbf{D}}\left(\psi\left(z_{1}\right), \psi\left(z_{2}\right)\right) \leq 1$, then $\rho_{\Omega}\left(z_{1}, z_{2}\right) \leq C$.

Proof. Choose a geodesic between the image points and a point $w$ where this crosses the image of $\gamma$. Let $w_{1}, w_{2}$ be the preimages of this point under the continuous boundary values of $\psi$ restricted to $\Omega_{1}$ and $\Omega_{2}$ respectively. Then $\rho_{\Omega}\left(w_{1}, z_{1}\right) \leq C$ since $\psi$ is a quasi-isometry on $\Omega$. Similar for $w_{2}$ and $z_{2}$. Finally, $\rho\left(w_{1}, w_{2}\right) \leq C$ by Lemma 4.1.

Lemma 4.3. The map $\psi: \Omega \rightarrow \mathbf{D}$ is a quasi-isometry between the hyperbolic metrics.

Proof. It is enough to show that the forward image of a hyperbolic unit ball has bounded diameter and that the inverse image of a unit ball has bounded diameter.

The forward image is easy to deal with. If $D$ has diameter 1 , then it can hit only a bounded number of distinct decomposition pieces, since there a lower bound on the hyperbolic distance between distinct crosscut (Lemma 3.1). Moreover, where $D$ meets a crosscut, the map on different sides of the crosscut differ by a bounded amount (Lemma 4.1). Moreover, the intersection $D$ with each piece has bounded diameter by Lemma 3.5. Thus the image is a bounded number of bounded sets, each of which is a bounded distance from its neighbors. Thus the image has uniformly bounded diameter.

For the other direction, first let $C_{1}=C(1)$ in Corollary 3.4. Let $C_{2} \geq 1$ be the constant from Corollary 4.2 and let $C_{3}$ be the quasi-isometry constant from Lemma 3.6. Let $C_{4}$ be the constant $C$ from Lemma 2.1. Now choose $M>8 C_{1}+C_{2} C_{3}\left(C_{4}+8\right)$.

We need two facts about hyperbolic geometry in the disk:

Lemma 4.4. For any $M>0$ there is an $\epsilon>0$ so that if two disjoint infinite geodesics $\gamma_{1}, \gamma_{2}$ in $\mathbf{D}$ both hit the disk $D_{\rho}(0, \epsilon)=\{z: \rho(0, z)<\epsilon\}$, then the segments $\gamma_{1} \cap D_{\rho}(0, M)$ and $\gamma_{2} \cap D_{\rho}(0, M)$ each lie in a hyperbolic 1-neighborhood of the other.

Lemma 4.5. There is a $r>0$ so that if $\gamma_{1}, \gamma_{2}, \gamma_{3}$ are three disjoint infinite geodesics in $\mathbf{D}$ so that none of them separates the other two, then no hyperbolic $r$-disk can intersect all three geodesics.

Both of these follow from some elementary hyperbolic trigonometry. Suppose a hyperbolic triangle has geodesic segments of lengths $a, b, c$ for edges with opposite angles of $\alpha, \beta, \gamma$ respectively. The first cosine rule says,

$$
\cosh c=\cosh a \cosh b-\sinh a \sinh b \cos \gamma
$$

and the second cosine rule says

$$
\cosh c=\frac{\cos \alpha \cos \beta+\cos \gamma}{\sin \alpha \sin \beta}
$$


See page 148 of Beardon's book [3].

Proof of Lemma 4.4. Consider the left side of Figure 7, which shows hyperbolic disks of radii $\epsilon$ and $M$ around 0 , the geodesic $\gamma_{1}$ through 0 connecting -1 and 1 , and a second geodesic $\gamma_{2}$ starting at -1 and tangent to the ball of radius $\epsilon$. This geodesic has the greatest divergence from $\gamma_{1}$ among those we are considering, so we want to compute how far apart these geodesics are at the points $x, y$ where they leave the $M$-disk near 1 . The origin is connected to $\gamma_{2}$ by a radial segment of hyperbolic length $\epsilon$ and making an angle $\theta=\frac{\pi}{2}-\delta$ with $[-1,0]$ and and angle $\pi / 2$ with $\gamma_{2}$. By the second cosine rule (applied with angles $0, \theta, \theta$ ),

$$
\cosh \epsilon=\frac{\cos \theta \cos \pi / 2+1}{\sin \theta \sin \pi / 2} \leq \frac{1}{\sin \left(\frac{\pi}{2}-\delta\right)} \leq 1+\delta^{2},
$$

if $\delta$ is small enough. Thus $\epsilon \leq 2 \delta$ if $\delta$ is small enough. Now consider the hyperbolic triangle with vertices $0, x, y$. It has two sides of hyperbolic length $M$ meeting at an angle $\phi>\frac{\pi}{2}-\theta=\delta$ (because the angle marked $\tau$ must be $<\frac{\pi}{2}$ ). Thus if $s$ is the hyperbolic distance from $x$ to $y$, the first cosine rule implies,

$$
\cosh s=\cosh ^{2} M-\sinh ^{2} M \cos \phi=1+\sinh ^{2} M(1-\cos \phi) \leq 1+\frac{1}{2} \delta^{2} \sinh ^{2} M .
$$

Thus $s \leq \frac{1}{2}$ if $\delta \leq\left(2\left(\cosh \left(\frac{1}{2}\right)-1\right) \sinh ^{-2} M\right)^{1 / 2} \leq \sqrt{2} e^{-M}$ for $M$ large. This proves that any geodesic hitting the $\epsilon$-disk (with $\epsilon \leq e^{-M}$ ) around 0 and disjoint from $\gamma_{1}$ stays within hyperbolic distance $\frac{1}{2}$ of $\gamma_{1}$ as long as it is inside the $M$-disk around 0 . This proves the lemma.
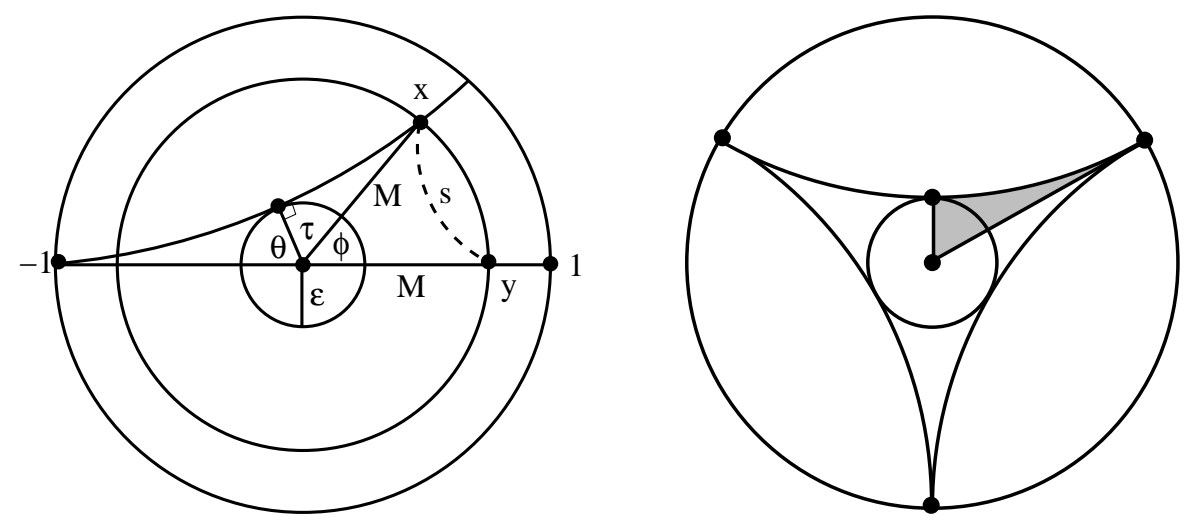

Figure 7. The proofs of Lemmas 4.4 and 4.5. On the left we show that disjoint geodesics that come close must stay close for a long time. On the right we show that three disjoint geodesics, none of which separate the other two, cannot all come close to the same point.

Proof of Lemma 4.5. The smallest disk that hits three non-separating geodesics will have minimal radius $r$ when the geodesics form an ideal triangle as on the right side Figure 7. Assume the disk is centered at 0 and connect 0 to closest point of one of the sides and to the endpoint of this side, forming a triangle with angles $0, \pi / 2, \pi / 3$. The second cosine rule says

$$
\cosh r=\frac{\cos \frac{\pi}{2} \cos \frac{\pi}{3}+\cos 0}{\sin \frac{\pi}{2} \sin \frac{\pi}{3}}=\frac{2}{\sqrt{3}}>1,
$$

which implies $r>0$. 
Now we continue with the proof of Lemma 4.3. With $M$ as above, choose $\epsilon>0$ as in Lemma 4.4. Without loss of generality, we also assume $\epsilon<\delta$ where $\delta$ is from Lemma 4.5. We claim that at most a bounded number of pieces can have images which hit such an $\epsilon$-ball $D$. This will prove the lemma (since if we can bound the number of image pieces hitting an $\epsilon$-ball we can bound the number hitting a unit ball by covering it by $\epsilon$-balls).

Suppose $N+1$ pieces do. By out choice of $\epsilon<\delta$ and Lemma 4.5, we can assume the image domains $\Omega_{1}, \ldots, \Omega_{N+1}$ and for a chain where each domain is adjacent to its successor. Then there are $N$ crosscuts whose images are geodesics that hit $D$. By our choice of $\epsilon$ there are unit balls $D_{1}, D_{2}$ each distance $M$ from $D$ which hits all $N$ geodesics. See Figure 8. By Corollary 4.2, the preimages of these two balls each have hyperbolic diameter which is $C_{2} N$ and their distance apart is $\geq M / C_{3}$. For each piece $\Omega_{k}$, choose a pair of points, one each in the preimages of $D_{1}, D_{2}$ intersected with $\Omega_{k}$ and consider the geodesic between them.

We will prove $N \leq C_{1}$. Choose a connected subchain of $K$ domains with $K<$ $2 C_{1} \leq M / 4$. Then by our choice of $M$ and Lemma 2.1 , there is a single point $z$ so that all $K$ geodesics pass within $C_{4} \exp \left(-C_{2}\left(C_{4}+8\right) / 8\right) \leq 1$ of $z$. This means $K \leq C_{1}$. But if all subchains have length $\leq C_{1}$ then $N \leq C_{1}$, as claimed. This proves the lemma.

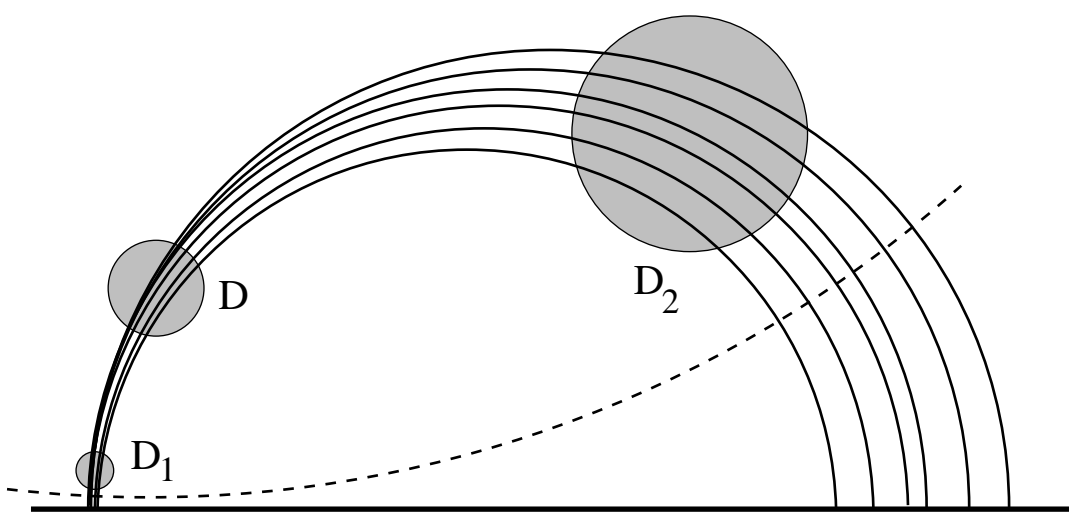

Figure 8. If $N$ pieces have images that hit an $\epsilon$-ball $D$, then they must also hit unit balls that are $M \gg N$ apart. The preimages have diameter $O(N)$ and geodesics between the two preimages must all hit a fixed unit ball, which limits the number of possible geodesics (since they are uniformly separated). The dashed line indicates the $M$-ball concentric with $D$.

\section{Iterating to a conformal map}

Suppose $\Omega$ is a simply connected plane domain bounded by a polygonal curve. The Schwarz-Christoffel formula gives the general form of a conformal map $f: \mathbf{D} \rightarrow$ $\Omega$ as

$$
f(z)=A+C \int^{z} \prod_{k=1}^{n}\left(1-\frac{w}{z_{k}}\right)^{\alpha_{k}-1} d w
$$

where $\alpha \pi=\left\{\alpha_{1} \pi, \ldots, \alpha_{n} \pi\right\}$, are the interior angles at the vertices $\mathbf{v}=\left\{v_{1}, \ldots, v_{n}\right\}$, and $\mathbf{z}=\left\{z_{1}, \ldots, z_{n}\right\}=f^{-1}(\mathbf{v})$ are the conformal preimages of the vertices (also know as the Schwartz-Christoffel parameters). We know the angles, so finding the conformal map is equivalent to finding the SC-parameters. For a fixed $\alpha$, we can 
think of the Schwartz-Christoffel formula as giving a map $S$ from $n$-tuples on the circle to $n$-tuples of complex numbers.

Given a map $G$ going from polygons to $n$-tuples, we can compose it with $S$ to get a map $F=G \circ S$ mapping $n$-tuples to $n$-tuples and the desired parameters $\mathbf{z}_{*}$ are a solution to $F(\mathbf{z})=\mathbf{z}_{0}=G(P)$, where $P$ is the target polygon. If $G$ is a good approximation to the inverse of $S$, then $F$ should be close to the identity and we can try to solve this equation by the iteration

$$
\left.\mathbf{z}_{0}=L(\Omega), \quad \mathbf{z}_{n+1}=\mathbf{z}_{n}-\left(F\left(\mathbf{z}_{n}\right)-\mathbf{z}_{0}\right)\right) .
$$

In [11] Driscoll and Vavasis define a map $G$ using cross ratios and the Delaunay triangulation and call this iteration "simple CRDT". Note that (5.1) is a special case of

$$
\mathbf{z}_{n+1}=\mathbf{z}_{n}-A^{-1}\left(F\left(\mathbf{z}_{n}\right)-\mathbf{z}_{n}\right),
$$

when $A$ is the identity matrix. If $A$ is the Jacobian of the function $F(\mathbf{z})-\mathbf{z}_{0}$, then this is Newton's method for several variables. However, it may be expensive to compute or even approximate the Jacobian. A compromise is to start the iteration with $A$ equal to the identity matrix and use a Broyden update to modify $A$ at each step. (A Broyden update multiplies the current matrix $A$ by a rank one matrix based on the on our most recent evaluation of $F$; the details are described in Chapter 8 of [10]). The CRDT iteration with Newton's method is called "full CRDT" in [11]; the version with Broyden updates is called "shortcut CRDT".

Theorem 1.1 says that a treelike decomposition of $\Omega$ gives rise to a piecewise length preserving mapping $G: \partial \Omega \rightarrow \partial \mathbf{D}$ which is only a bounded distance away from the Riemann mapping in a quasiconformal sense. If we compose our two maps $F=G \circ S$, we get a mapping of $n$-tuples to $n$-tuples and Theorem 1.1 roughly says that points are moved only a bounded distance by this map, at least for the distance

$$
d_{Q C}(\mathbf{w}, \mathbf{z})=\inf \{\log K: \exists K \text {-quasiconformal } h: \mathbf{D} \rightarrow \mathbf{D} \text { such that } h(\mathbf{z})=\mathbf{w}\} .
$$

Thus on large scales, composition looks like the identity and we can hope that (5.1) and (5.2) will converge. When the decomposition is trivial (just one piece equal to all of $\Omega$ ), iteration (5.1) is just Davis's method [9], [2]. If the decomposition is the Delaunay triangulation, the resulting iteration is similar to CRDT (but not quite the same; we will refer to it as ELDT for Edge Lengths and Delaunay Triangulations). As with CRDT we can define "simple", "full" or "shortcut" ELDT, although these methods do not seem to do quite as well as the CRDT variants.

See Figures 9 and 10 for a comparison of the methods for one example. Figure 9 shows the polygon, its Delaunay triangulation and two intermediate decompositions. Figure 10 show that the Schwarz-Christoffel images corresponding to the first ten iterations of shortcut ELDT (this uses Broyden updates starting from the identity).

In Figure 10, we plot $-\log (K-1)$ where $K$ is an upper bound for the quasiconformal error at each step, and the PLP maps corresponding to the three decompositions in Figure 9. The method for estimating the upper bound $K$ is as follows. Suppose $P_{0}$ is the target polygon and $P_{n}=S\left(\mathbf{z}_{n}\right)$. For each triangle $T$ in the Delaunay triangulation of $P_{0}$ compute the affine map from $T$ to the corresponding triangle $T^{\prime}$ in $P_{n}$. More explicitly, use a conformal linear map to send each triangle to one of the form $\{0,1, a\}$ and $\{0,1, b\}$. The affine map which fixes 0 and 1 and sends $a$ to $b$ is of the form $f(z) \rightarrow \alpha z+\beta \bar{z}$ where $\alpha+\beta=1$ and $\beta=(b-a) /(a-\bar{a})$ and from this it 
is easy to compute that

$$
K_{f}=\frac{1+\left|\mu_{f}\right|}{1-\left|\mu_{f}\right|}, \text { where } \mu_{f}=\frac{f_{\bar{z}}}{f_{z}}=\frac{\beta}{\alpha}=\frac{b-a}{b-\bar{a}} .
$$

If the triangle $T^{\prime}$ is degenerate, or has the opposite orientation as $T$, we simply give $\infty$ as our QC bound $K$. The maps on each triangle fit together to give a $\mathrm{QC}$ map between $P_{0}$ and $P_{n}$. Transferring this map back to the disk by conformal maps gives a QC map of the disk to itself which sends the true parameters to current guess. This gives a rigorous upper bound on the QC distance between $\mathbf{z}_{*}$ and $\mathbf{z}_{n}$, even though we do not know $\mathbf{z}_{*}$.
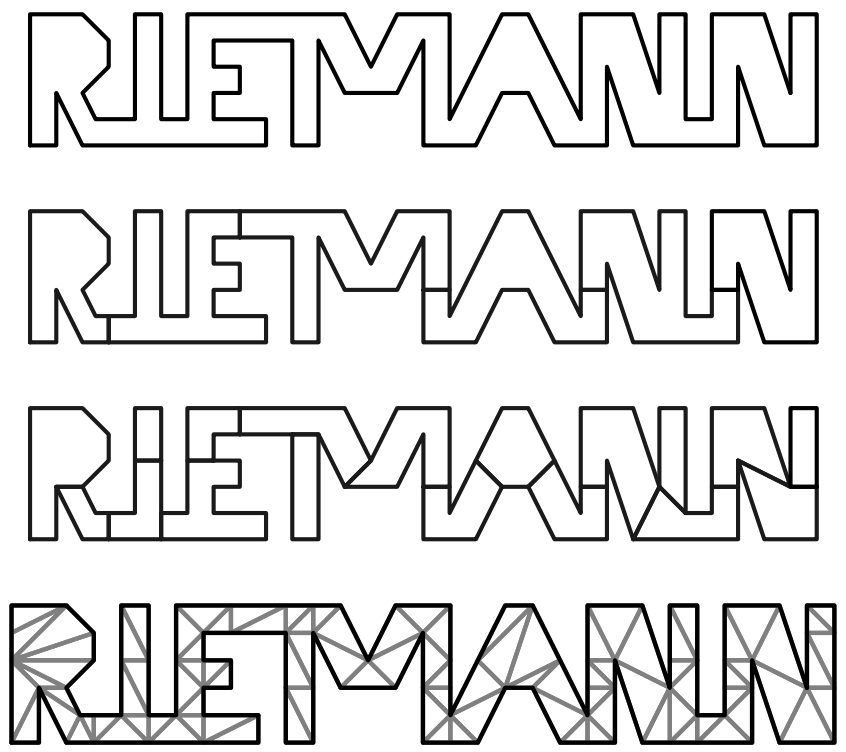

Figure 9. A 98-gon and three tree-like decompositions, including the Delaunay triangulation on the bottom.

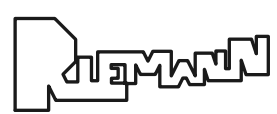

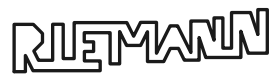

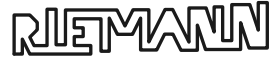

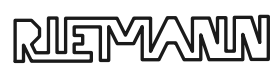

Rñ
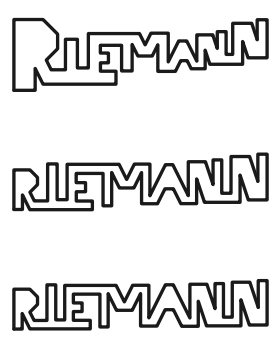

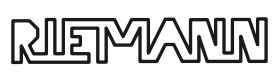

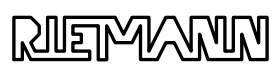

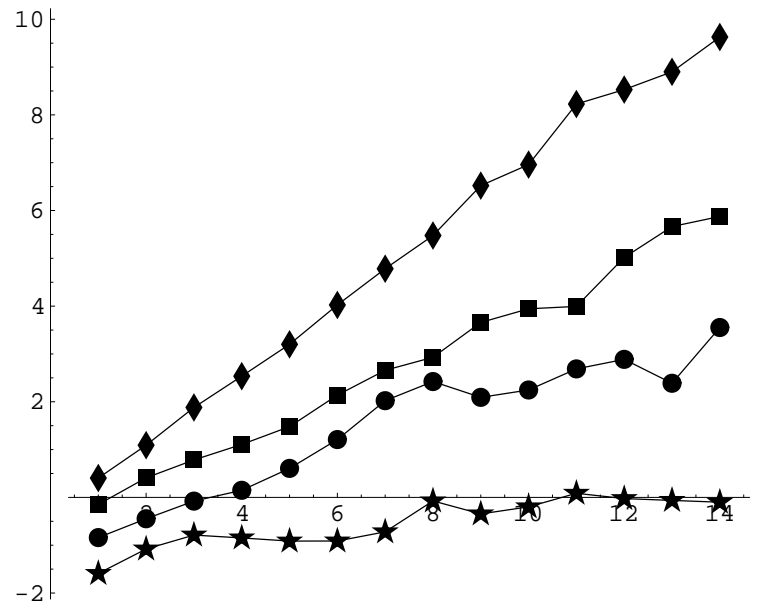

Figure 10. On the left are the first ten iterations of "shortcut ELDT". On the right is the plot of $-\log (K-1)$ for 15 iterations using Broyden updates and $G$ corresponding to the CRDT map (diamonds) and the PLP maps for the three decompositions in Figure 9. CRDT is the best and the PLP maps do better as the decomposition becomes finer. The left picture corresponds to first 10 points plotted with squares. 


\section{References}

[1] Ahlfors, L. V.: Lectures on quasiconformal mappings. - Univ. Lecture Ser. 38, with supplemental chapters by C. J. Earle, I. Kra, M. Shishikura and J. H. Hubbard, Amer. Math. Soc., Providence, RI, second edition, 2006.

[2] Banjai, L., and L. N. Trefethen: A multipole method for Schwarz-Christoffel mapping of polygons with thousands of sides. - SIAM J. Sci. Comput. 25:3, 2003, 1042-1065 (electronic).

[3] Beardon, A. F.: The geometry of discrete groups. - Springer-Verlag, New York, 1983.

[4] Bishop, C. J.: Bounds for the CRDT conformal mapping algorithm. - Comput. Methods Funct. Theory 10:1, 2010, 325-366.

[5] Bishop, C. J.: Conformal mapping in linear time. - Discrete Comput. Geom. (to appear).

[6] Bishop, C. J.: Estimates for harmonic conjugation. - Preprint, 2009.

[7] Bishop, C. J.: A fast QC-mapping theorem for polygons. - Preprint, 2009.

[8] Bishop, C. J.: Treelike decompositions of simply connected domains. - Preprint, 2009.

[9] DAVIS, R. T.: Numerical methods for coordinate generation based on Schwarz-Christoffel transformations. - In: Proceedings of the 4th AIAA Computational Fluid Dynamics Conference, Williamsburg, VA, 1979, 1-15.

[10] Dennis, J. E., JR., and R. B. Schnabel: Numerical methods for unconstrained optimization and nonlinear equations. - Prentice Hall Series in Computational Mathematics, Prentice Hall, Englewood Cliffs, NJ, 1983.

[11] Driscoll, T.A., and S. A. Vavasis: Numerical conformal mapping using cross-ratios and Delaunay triangulation. - SIAM J. Sci. Comput. 19:6, 1988, 1783-1803 (electronic).

[12] Epstein, D. B. A., A. Marden, and V. Markovic: Quasiconformal homeomorphisms and the convex hull boundary. - Ann. of Math. (2) 159:1, 2004, 305-336.

[13] Garnett, J. B., and D. E. Marshall: Harmonic measure. - New Math. Monogr. 2, Cambridge Univ. Press, Cambridge, 2005.

[14] Gehring, F. W., and W. K. Hayman: An inequality in the theory of conformal mapping. J. Math. Pures Appl. (9) 41, 1962, 353-361.

[15] Ghamsari, M.: Extension domains. - PhD thesis, Univ. of Michigan, 1990.

[16] Pommerenke, Ch.: One-sided smoothness conditions and conformal mapping. - J. London Math. Soc. (2) 26:1, 1982, 77-88.

[17] VäIsÄLÄ, J.: Homeomorphisms of bounded length distortion. - Ann. Acad. Sci. Fenn. Ser. A I Math. 12:2, 1987, 303-312.

[18] VÄıs̈̈lä, J.: Free quasiconformality in Banach spaces. II. - Ann. Acad. Sci. Fenn. Ser. A I Math. 16:2, 1991, 255-310. 